\title{
Spherically symmetric potential in noncommutative spacetime with a compactified extra dimensions
}

\author{
Sêcloka Lazare Guedezounme ${ }^{1, a}$, Antonin Danvidé Kanfon ${ }^{1,2, b}$, Dine Ousmane Samary ${ }^{1,2,3, c}$ \\ ${ }^{1}$ International Chair in Mathematical Physics and Applications (ICMPA-UNESCO Chair), University of Abomey-Calavi, 072B.P.50, Cotonou, \\ Republic of Benin \\ ${ }^{2}$ Faculté des Sciences et Techniques, University of d'Abomey-Calavi, Cotonou, Benin \\ ${ }^{3}$ Max Planck Institute for Gravitational Physics, Albert Einstein Institute, Am Mühlenberg 1, 14476 Potsdam, Germany
}

Received: 2 May 2016 / Accepted: 8 September 2016 / Published online: 15 September 2016

(c) The Author(s) 2016. This article is published with open access at Springerlink.com

\begin{abstract}
The Schrödinger equation of the spherically symmetrical quantum models such as the hydrogen atom problem seems to be analytically non-solvable in higher dimensions. When we try compactifying one or several dimensions this question can maybe solved. This paper presents a study of the spherically symmetrical quantum models on noncommutative spacetime with compactified extra dimensions. We provide analytically the resulting spectrum of the hydrogen atom and Yukawa problem in $4+1$ dimensional noncommutative spacetime in the first order approximation of the noncommutative parameter. The case of higher dimensions $D \geq 4$ is also discussed.
\end{abstract}

\section{Introduction}

One of the recently discovered concepts that has impacted the theoretical physics community in the most significant way is most likely the idea of a noncommutative (NC) spacetime, which led to a NC generalization of quantum mechanics and field theory. The idea of noncommutativity of spacetime was first discussed in the work by Snyder [1] and Connes [2,3]. The above concept (NC) spacetime allows one to find a possible solution to ultraviolet divergencies in quantum field theory $[4,5]$. The NC physics also arises as a possible scenario for the short-distance behavior of physical theories (at the Planck scale). At this scale, the universal constants $c, \hbar$, and $G$ appear naturally equivalent. Below the Planck length, the distance loses its meaning [4-6] and the physical phenomena are believed to be nonlocal. NC geometry could be realized by introducing the noncommutativity through the coordinates

\footnotetext{
a e-mail: guesel10@yahoo.fr

be-mail: kanfon@yahoo.fr

${ }^{c}$ e-mail: dine.ousmane.samary@aei.mpg.de
}

which satisfy the commutation relations $\left[x^{\mu}, x^{\nu}\right]=i \theta^{\mu \nu}$, where $\theta^{\mu \nu}$ is a skew-symmetric matrix characterizing the deformation of the spacetime. This leads to a new Heisenberg uncertainty relation, given on the spacetime coordinates by $\Delta x^{\mu} \Delta x^{\nu} \geq \theta^{\mu \nu}$, and this makes this spacetime a quantum space [6,7]. The important implications of noncommutativity are the loss of Lorentz invariance in the dispersion relations and the loss of causality [8-13]. Intuitive arguments involving quantum mechanics in $\mathrm{NC}$ space are realized by imposing the commutation relations, now between coordinates and momenta, as

$$
\left[x^{\mu}, x^{\nu}\right]=i \theta^{\mu \nu}, \quad\left[p^{\mu}, p^{\nu}\right]=i \gamma^{\mu \nu}, \quad\left[x^{\mu}, p^{\nu}\right]=i \hbar \kappa^{\mu \nu}
$$

where $\gamma^{\mu \nu}$ are also skew-symmetric matrices. In this paper we restrict ourself to the case where $\gamma^{\mu \nu}=0$, this implies that $\kappa^{\mu \nu}=\delta^{\mu \nu}$, the Kronecker symbol. We also assume that the tensor $\theta^{\mu \nu}$ is chosen to have the dimension of length . time, i.e., $\theta^{0 j}=\theta^{j} \in \mathbb{R}, \theta^{i j}=0, \quad i, j=1,2, \ldots, D$. The noncommutative variables can be expressed in terms of commutative coordinates as $x^{j}=x_{c}^{j}-i \theta^{j} \partial_{0}=(x)$, and $p^{j}=p_{c}^{j}, p^{0}=i \hbar \partial_{0}=E$, where the index $\mathrm{c}$ is used to specify the commutative variables and where $E$ is the energy of the system. The Hamiltonian of quantum system on NC space can be expressed with the commutative coordinates $H(x, p) \equiv H_{c}\left(x_{c}, p_{c}, \theta\right)$, where the parameter $\theta=\theta^{j}$ is showed to have the fundamental limit $\theta \preceq 1.6 \cdot 10^{-27} \mathrm{~m} \cdot \mathrm{s} \approx$ $0.3(\mathrm{keV})^{-2}$, which is smaller than the one obtained by the theory of quantum gravity $[14,15]$.

The compactified extra dimension is motivated by string theory, which predicts the existence of extra dimensions and noncommutativity between coordinates. Our idea is to understand how the eigenvalue problem changes if we periodically identify one of the NC coordinates $x^{j}=\left(x^{1}, x^{2}, x^{3}, x^{4}\right)$ in the target space, say $[-\pi R, \pi R] \ni w$, such that $x^{4}=$ 
$w-2 \pi R k, k \in \mathbb{Z}$, and $R$ is the radius of the circle. The wave function $\psi\left(x^{0}, x^{\bar{\ell}}, x^{4}\right), \bar{\ell}=1,2,3$, can be expanded in the Fourier mode as [16]

$\psi\left(x^{0}, x^{\bar{\ell}}, x^{4}\right)=\frac{1}{\sqrt{2 \pi R}} \sum_{n=-\infty}^{\infty} \psi_{n}\left(x^{0}, x^{\bar{\ell}}\right) \exp \left(\frac{i n x^{4}}{R}\right)$.

Note that the orthonormalized functions $(2 \pi R)^{-1 / 2} \exp$ $\left(i x^{4} n / R\right)$ are eigenfunctions of the operator $\nabla_{x^{4}}^{2}$ with eigenvalues $E_{n 0}=-n^{2} / R^{2}$. This means that the spectrum of a quantum system defined with one dimension compactified is in the form $E_{n l}=E_{n 0}+E_{n l}^{\prime}$, where $l$ is a positive integer, $E_{n l}^{\prime}$ depends on the potential associated to the system which is required to be computed. In the following paper we investigate the spectrum of the Coulomb and Yukawa Hamiltonian on $(4+1)$-dimensional NC spacetime. Using the first order approximation of the deformation parameter $\theta$ and by compactifying one extra dimension $x^{4}$ we have the resulting topology $\mathbb{R}^{3+1} \times S^{1}$ (see [17] and [18] for the essential reviews), the spectrum may be given exactly. We prove that in the case of space - time noncommutativity, the correction of the energy spectrum does not depend on the NC deformation parameter $\theta$ but rather on the parameter associated to the compactified dimension.

Our paper is organized as follows. In Sect. 2, we focus on the hydrogen atom in $(D+1)$-dimensional noncommutative space with non-compactified extra dimension. We discuss the particular case where $D=4$ in which the solution of the spectral problem can be solved. The Yukawa potential is also discussed in this section. In Sect. 3 the same problem is solved with compactified extra dimensions. The discussion and conclusion are given in Sect. 4.

\section{Hydrogen atom in noncommutative space with non-compactified extra dimension}

In this section we focus on the hydrogen atom problem defined in $(D+1)$ dimensional NC spacetime (we consider the particular case where $D=4$ ). To be specific, the model is given with the spherical potential of the form

$V\left(\vec{r}_{n c}\right)=-\frac{q_{e}^{2}}{\left|\vec{r}_{n c}\right|^{D-2}}$,

where $q_{e}$ is related to the atomic charge and where we use the following notation: $\vec{r}_{n c}=\vec{r}-i \vec{\theta} \partial_{0}$, i.e., the NC coordinates are $\vec{r}_{n c}=(x)$ and the commutative coordinates are $\vec{r}=$ $\left(x_{c}\right)$. It would be advisable to work in a spherical coordinates system, $\vec{r}=\left(r, \alpha_{1}, \alpha_{2}, \alpha_{3}\right)$, such that $r \in \mathbb{R}_{+}, 0<\alpha_{\bar{\ell}}<$ $\pi, \bar{\ell}=1,2$, and $0<\alpha_{3}<2 \pi$. It thus follows that the
Hamiltonian of the system is

$H=-\frac{\hbar^{2}}{2 m}\left[\frac{\partial^{2}}{\partial r^{2}}+\frac{D-1}{r} \frac{\partial}{\partial r}-\frac{\mathcal{L}^{2}(D-1)}{r^{2}}\right]+V\left(\vec{r}_{n c}\right)$,

where $\mathcal{L}^{2}(D-1)$ is the Laplace-Beltrami operator on the $(D-1)$-sphere. Hence the potential (3), using the first order Taylor expansion on $\vec{\theta}$, is

$$
\begin{aligned}
V\left(\vec{r}_{n c}\right) & =-\frac{q_{e}^{2}}{\left|\vec{r}-i \vec{\theta} \partial_{0}\right|^{D-2}} \\
& \approx-\frac{q_{e}^{2}}{r^{D-2}}\left(1+i(D-2) \frac{\vec{r} \cdot \vec{\theta}}{r^{2}} \partial_{0}\right) .
\end{aligned}
$$

We consider the adequate choice, such that the vector $\vec{\theta}$ is transformed in the spherical coordinates to $\vec{r} \cdot \vec{\theta} \equiv r \theta$ [14]. Furthermore, the spherical functions $\mathcal{Y}_{\ell}^{(D-1)}\left(\alpha_{1}, \alpha_{2}, \ldots\right.$, $\left.\alpha_{D-1}\right)$, which are the eigenfunctions of the operator $\mathcal{L}(D-1)$ are considered:

$\mathcal{L}^{2}(D-1) \mathcal{Y}_{\ell}^{(D-1)}=\ell(\ell+D-2) \mathcal{Y}_{\ell}^{(D-1)}=\lambda_{D} \mathcal{Y}_{\ell}^{(D-1)}$,

where $\ell$ is the orbital angular momentum quantum number.

Note that the Hamiltonian (4) depends on the partial derivative with respect to the time $t$, due to the relation (5). But one can show that the wave function $\psi(\vec{r}, t)$, namely the solution of the Schrödinger equation is expressed as $\psi(\vec{r}, t)=\mathcal{Y}_{\ell}^{(D-1)} \psi(r) f(t)$, where the time dependent function is $f(t)=\exp \left(-\frac{i}{\hbar} E t\right)$ and where $\psi(r)$ satisfies the radial equation

$\left[\frac{\mathrm{d}^{2}}{\mathrm{~d} r^{2}}+\frac{D-1}{r} \frac{\mathrm{d}}{\mathrm{d} r}-\left(\frac{\lambda_{D}}{r^{2}}-\frac{v^{2}}{r^{D-2}}-\frac{\mu_{D}}{r^{D-1}}-\alpha^{2}\right)\right] \psi(r)=0$,

with $\alpha^{2}=\frac{2 m E}{\hbar^{2}}, v^{2}=\frac{2 m q_{e}^{2}}{\hbar^{2}}, \mu_{D}=(D-2) v^{2} \theta E / \hbar$. Now for $D=4$, this equation turns to become

$$
\left[\frac{\mathrm{d}^{2}}{\mathrm{~d} r^{2}}+\frac{3}{r} \frac{\mathrm{d}}{\mathrm{d} r}-\left(\frac{\lambda_{4}-v^{2}}{r^{2}}-\frac{\mu_{4}}{r^{3}}-\alpha^{2}\right)\right] \psi(r)=0 .
$$

However, in this case (unlike for the commutative case discussed in $[17,18])$, such an equation seems to be nonsolvable. We provide an algebraic method, which will allow us to derive the solution of this equation. For this purpose, let us reparameterize the function $\psi(r)$ as $\psi(r):=\psi(r, \theta)$. Then $\psi(r, 0)$ corresponds to the solution of Eq. (8) in the case where $\theta=0$. The first order Taylor expansion on $\theta$ of the function $\psi(r, \theta)$ takes the form

$\psi(r, \theta)=\psi(r, 0)+\left.\theta \frac{\mathrm{d} \psi(r, \theta)}{\mathrm{d} \theta}\right|_{\theta=0}+\mathcal{O}\left(\theta^{2}\right)$.

We get simply

$\psi(r, 0)=\frac{c}{r} J_{v}(\alpha r)+\frac{c^{\prime}}{r} Y_{v}(\alpha r), \quad c, c^{\prime} \in \mathbb{R}$, 
where $J_{v}(\alpha r)$ and $Y_{v}(\alpha r)$ are, respectively, the first and second kind Bessel functions (see [18] for more details). By replacing the solution (9) in the partial differential equation (8), we get

$\left[\frac{\mathrm{d}^{2}}{\mathrm{~d} r^{2}}+\frac{3}{r} \frac{\mathrm{d}}{\mathrm{d} r}+\left(\alpha^{2}-\frac{\lambda_{4}-v^{2}}{r^{2}}\right)\right] \tilde{\chi}(r)=-\frac{2 v^{2} E}{\hbar r^{3}} \psi(r, 0)$,

where $\tilde{\chi}(r)=\left.\frac{\mathrm{d} \psi(r, \theta)}{\mathrm{d} \theta}\right|_{\theta=0}$. This equation corresponds to a nonhomogeneous differential equation, which can be solved easily. For $\epsilon=\left(1+\lambda_{4}-v^{2}\right)^{1 / 2}$ and $g(r)=$ $-2 v^{2} E \psi(r, 0) / \hbar r^{3}$, by using the Wronskian method, the solution of Eq. (11) takes the form

$$
\begin{aligned}
\tilde{\chi}(r)= & c J_{\epsilon}(\alpha r)+c^{\prime} Y_{\epsilon}(\alpha r) \\
& -\frac{\pi}{2 r} J_{\epsilon}(\alpha r) \int_{1}^{r} x^{2} Y_{\epsilon}(\alpha x) g(x) \mathrm{d} x \\
& +\frac{\pi}{2 r} Y_{\epsilon}(\alpha r) \int_{1}^{r} x^{2} J_{\epsilon}(\alpha x) g(x) \mathrm{d} x, c, c^{\prime} \in \mathbb{R} .
\end{aligned}
$$

Note that there are some difficulties, however. One defect of this method (in the commutative and $\mathrm{NC}$ case) is that the energy spectrum can only be determined numerically, and we do not deal here with a numerical method to provide this spectrum. In more than $(4+1)$ dimensions, the differential equations (7) are much more complicated.

Now let us discuss the case of a Yukawa potential:

$V\left(\vec{r}_{n c}\right)=-V_{0} \frac{e^{-\eta r_{n c}}}{\left|\vec{r}_{n c}\right|^{D-2}}$,

where $V_{0}$ and $\eta$ depend on the constant of the neutral atom. In order to probe this potential, we write the expression (13) at the first order in $\theta$ as

$V\left(\vec{r}_{n c}, t\right)=-\frac{V_{0} e^{-\eta r}}{r^{D-2}}\left[1+i(\eta r+D-2) \frac{\theta}{r} \partial_{0}\right]$.

After separation variables in the Schrödinger equation, it become easy to show that the radial equation is given by the following:

$$
\left[\frac{\partial^{2}}{\partial r^{2}}+\frac{D-1}{r} \frac{\partial}{\partial r}+s_{D}(r)\right] \psi(r)=0 .
$$

where

$$
\begin{aligned}
s_{D}(r)= & \frac{2 m V_{0} e^{-\eta r}}{\hbar^{3} r^{D-2}}(D-2+\eta r) \frac{E \theta}{r} \\
& +\frac{2 m E}{\hbar^{2}}-\frac{\lambda_{D}}{r^{2}}+\frac{2 m V_{0} e^{-\eta r}}{\hbar^{2} r^{D-2}},
\end{aligned}
$$

In the particular case where $D=4$, this equation is reduced to

$$
\frac{\mathrm{d}^{2} \psi(r)}{\mathrm{d} r^{2}}+\frac{3 \mathrm{~d} \psi(r)}{r} \frac{\mathrm{d} r}{\mathrm{~d} r}(r) \psi(r)=0 .
$$

This equation (including now the occurrence of the exponential factor $e^{-\eta r}$ ) has the same shape as (8), and therefore the same conclusion with (12) will be made.

\section{Hydrogen atom in noncommutative space with compactified extra dimension}

In this section we consider $(D+1) \mathrm{NC}$ spacetime, where one dimension $x^{D}$ is compactified on a circle of radius $R$. This means that $\mathbb{R}^{D+1}$ is reduced to $\mathbb{R}^{D-1+1} \times[-\pi R, \pi R]$ and $x^{D}=\omega-2 \pi n R, n \in \mathbb{Z}$. The interaction potential (5) written now with the required coordinates $\vec{r}=\left(r, \alpha_{1}, \ldots, \alpha_{D-2}\right)$ and the compactify coordinate $w$ is

$$
\begin{aligned}
V(\vec{r}, w)= & -q_{e}^{2} \sum_{n=-\infty}^{\infty}\left\{\frac{1}{\left(r^{2}+(w-2 \pi n R)^{2}\right)^{\frac{D-2}{2}}}\right. \\
& \left.+\frac{i(D-2) r \theta}{\left(r^{2}+(w-2 \pi n R)^{2}\right)^{\frac{D}{2}}} \partial_{0}\right\},
\end{aligned}
$$

where $r$ is now the radial coordinates in $(D-1)$-dimensional space, and the extra dimension $x^{D}$ satisfy the condition $\mid x^{D}$ $2 \pi n R \mid \leq \pi R$. For $D=4$, we get

$$
\begin{aligned}
V(\vec{r}, w)= & -q_{e}^{2} \sum_{n \in \mathbb{Z}}\left\{\frac{1}{r^{2}+(w-2 \pi n R)^{2}}\right. \\
& \left.+\frac{2 i r \theta}{\left(r^{2}+(w-2 \pi n R)^{2}\right)^{2}} \partial_{0}\right\} .
\end{aligned}
$$

Then we can compute the following identities:

$$
\sum_{n \in \mathbb{Z}} \frac{1}{r^{2}+(w-2 \pi n R)^{2}}=\frac{1}{2 R r} \frac{\sinh (r / R)}{\cosh (r / R)-\cos (w / R)}
$$

and

$$
\sum_{n \in \mathbb{Z}} \frac{2 r}{\left(r^{2}+(w-2 \pi n R)^{2}\right)^{2}}=G(r)+F(r)
$$

where

$$
\begin{aligned}
& G(r)=-\frac{1}{2 R r^{2}} \frac{\sinh (r / R)}{\cosh (r / R)-\cos (w / R)}, \\
& F(r)=\frac{1}{2 R^{2} r} \frac{1-\cosh (r / R) \cos (w / R)}{(\cosh (r / R)-\cos (w / R))^{2}} .
\end{aligned}
$$

The potential $V(\vec{r}, w)$ is periodic with respect to the $w$ direction, and it can be expanded in a Fourier series as follows: 
$V(\vec{r}, w)=\sum_{n \in \mathbb{Z}} a_{n}(r) e^{i n w / R}+i \theta \sum_{n \in \mathbb{Z}} b_{n}(r) e^{i n w / R} \partial_{0}$,

where

$$
\begin{aligned}
& a_{n}(r)=-\frac{q_{e}^{2}}{2 r R} e^{-|n| r / R}, \\
& b_{n}(r)=-\frac{q_{e}^{2}}{2 R r^{2}}[1+|n|] e^{-|n| r / R},
\end{aligned}
$$

such that

$V(\vec{r}, w)=-\frac{q_{e}^{2}}{2 r R} \sum_{n \in \mathbb{Z}}\left[1+i \theta\left(\frac{1}{r}+\frac{|n|}{R}\right) \partial_{0}\right] e^{-(|n| r-i n w) / R}$.

The separation of the variables in the Schrödinger equation shows that the radial function $\psi(r)$ satisfies

$\left(\frac{\mathrm{d}^{2}}{\mathrm{~d} r^{2}}+\frac{2}{r} \frac{\mathrm{d}}{\mathrm{d} r}-\frac{n^{2}}{R^{2}}+\alpha^{2}+\frac{\zeta}{r}-\frac{v_{\theta}^{2}}{r^{2}}\right) \psi_{n}(r)=0$,

with $\alpha^{2}=\frac{2 m E}{\hbar^{2}}, v_{\theta}^{2}=\lambda_{4}-\zeta E \theta / \hbar, \zeta=v^{2} /(2 R), v^{2}=$ $\frac{2 m q_{e}^{2}}{\hbar^{2}}$.

The solution of the above equation is expressed as

$$
\begin{aligned}
\psi_{n}(r)= & {\left[\frac{(2 \zeta)^{a+2} l !}{(2 l+1+a)^{a+3} \Gamma(l+1+a)}\right]^{\frac{1}{2}} r^{\frac{1}{2}(a-1)} e^{-\frac{\zeta r}{2 l+a+1}} } \\
& \times L_{l}^{a}\left(\frac{2 \zeta r}{2 l+a+1}\right), \quad a=\sqrt{4 v_{\theta}^{2}+1}
\end{aligned}
$$

where we have used the normalization condition $\int_{0}^{\infty} e^{-z} z^{a+1}$ $\left[L_{l}^{a}(z)\right]^{2} \mathrm{~d} z=\frac{(2 l+1+a) \Gamma(l+1+a)}{l !}$, and where $L_{l}^{a}$ stands for the generalized Laguerre polynomial. The quantum number $l$ is a positive integer, which corresponds to the physical situation. This integer is given by

$l=-\frac{1}{2}-\frac{1}{2} \sqrt{4 v_{\theta}^{2}+1}+\frac{\zeta R}{2 \sqrt{n^{2}-\alpha^{2} R^{2}}}$.

Two energy contributions appear from Eq. (28):

$E_{n l}^{(1)}=\frac{\hbar^{2}}{2 m}\left(\frac{n^{2}}{R^{2}}-\frac{\zeta^{2}}{\left(2 l+1+\sqrt{1+4 \lambda_{4}}\right)^{2}}\right)$

and

$$
\begin{aligned}
E_{n l}^{(2)}= & \frac{\hbar^{2}}{2 m} \frac{\zeta^{2}}{\left(2 l+1+\sqrt{1+4 \lambda_{4}}\right)^{2}} \\
& +\frac{\hbar \sqrt{1+4 \lambda_{4}}}{4 \theta \zeta}\left(2 l+1+\sqrt{1+4 \lambda_{4}}\right) .
\end{aligned}
$$

Let us discuss the energy spectrum (30). In the limit where $\theta \rightarrow 0, E_{n l}^{(2)}$ is not well defined. Also as expected in our introduction the eigenfunctions of the operator $\nabla_{x^{4}}^{2}$ with eigenvalues $E_{n 0}=-n^{2} / R^{2}$ are not recovered. Finally this expression cannot be taking into account as a solution of the eigenvalue problem. Then the energy spectrum becomes

$E_{n l}=\frac{\hbar^{2}}{2 m}\left(\frac{n^{2}}{R^{2}}-\frac{\zeta^{2}}{\left(2 l+1+\sqrt{1+4 \lambda_{4}}\right)^{2}}\right)$.
Remark 1 - Our result shows that the energy spectrum (31) does not depend on the $\mathrm{NC}$ parameter $\theta$ if we consider the first order approximation in this parameter. The solution of the eigenvalue problem of the hydrogen atom with one compactified dimension is solved numerically in [17, 18] (see also $[19,20]$ in the case where no dimensions are compactified). Due to the fact that $\lim _{\theta \rightarrow 0} E_{n l}=$ $E_{n l}$, the expression (31) can be considered as a solution of the hydrogen atom in $4+1$-dimensional spacetime for both the $\mathrm{NC}^{1}$ and the commutative cases, where one dimension is compactified.

- The quantity

$$
E_{n l}^{\prime}=-\frac{\hbar^{2} \zeta^{2}}{2 m\left(2 l+1+\sqrt{1+4 \lambda_{4}}\right)^{2}}
$$

corresponds to the reduced dimension energy spectrum and is discussed in the introduction of our paper.

We consider now the case of a Yukawa potential (13) for $D=4$. On shell, and with the compactified $x^{4}$ direction on the circle we get the reduced potential

$$
\begin{aligned}
V(\vec{r}, w)= & i V_{0} \theta\left[-\frac{1}{2 R r^{2}} \frac{\sinh (r / R)}{\cosh (r / R)-\cos (w / R)}\right. \\
& \left.+\frac{1}{2 R^{2} r} \frac{1-\cosh (r / R) \cos (w / R)}{(\cosh (r / R)-\cos (w / R))^{2}}\right] \partial_{0} \\
& -\frac{V_{0}}{2 R r} \frac{\sinh (r / R)}{\cosh (r / R)-\cos (w / R)} .
\end{aligned}
$$

Let us briefly give the proof of this relation. The goal of this proof is to compute the integral $\int_{\Gamma} f(z) \mathrm{d} z$, where $\Gamma$ is a closed contour on the complex plane and $f(z)$ is a holomorphic function given by

$$
f(z)=\frac{\cot (\pi z) \exp \left[-c\left((a-z)^{2}+b^{2}\right)^{1 / 2}\right]}{(a-z)^{2}+b^{2}},
$$

where $a, b, c$ are three real numbers. The poles of $f(z)$ are $z_{n}=n, n \in \mathbb{Z}, z_{I}=a+i b$, and $z_{I I}=a-i b$. Using the residue theorem,

$\int_{\Gamma} f(z) \mathrm{d} z=2 i \pi \sum \mathcal{R} \operatorname{es}[f(z)]=0$,

with

$$
\begin{aligned}
& \operatorname{Res}[f(z)]=\frac{\exp \left[-c\left((a-n)^{2}+b^{2}\right)^{\frac{1}{2}}\right]}{\pi\left[(a-n)^{2}+b^{2}\right]}, \\
& \underset{z_{n}}{\operatorname{Res}[f(z)]}=-\frac{i}{2 b} \cot [\pi(a+i b)], \quad \text { and }
\end{aligned}
$$

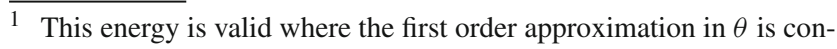
sidered. 


$$
\underset{z_{I I}}{\operatorname{Res}[f(z)]}=\frac{i}{2 b} \cot [\pi(a-i b)] .
$$

Hence,

$$
\begin{aligned}
& \sum_{n \in \mathbb{Z}} \frac{\exp \left[-c\left((a-n)^{2}+b^{2}\right)^{1 / 2}\right]}{(a-n)^{2}+b^{2}} \\
& =\frac{\pi}{b} \frac{\sinh (2 \pi b)}{\cosh (2 \pi b)-\cos (2 \pi a)} .
\end{aligned}
$$

The first term on the right hand side of (33) is

$$
\begin{gathered}
\sum_{n \in \mathbb{Z}} \frac{\exp \left[-\zeta\left(r^{2}+(w-2 \pi n R)^{2}\right)^{1 / 2}\right]}{r^{2}+(w-2 \pi n R)^{2}} \\
\quad=\frac{1}{2 R r} \frac{\sinh (r / R)}{\cosh (r / R)-\cos (w / R)} .
\end{gathered}
$$

The second term on the right hand side of Eq. (33) is the first order derivative of Eq. (38) respect to $r$. Then Eq. (33) is straightforwardly obtained.

Now using the fact that the function $V(\vec{r}, w)$ is periodic, the Fourier series can be given by

$V(\vec{r}, w)=\sum_{n \in \mathbb{Z}}\left(a_{n}(r)+i \theta b_{n}(r) \partial_{0}\right) e^{i n w / R}$,

where the Fourier coefficients are

$a_{n}(r)=-\frac{V_{0}}{2 r R} e^{-|n| r / R}$,

$b_{n}(r)=-V_{0}\left[\frac{1}{2 R r^{2}}+\frac{|n|}{2 R^{2} r}\right] e^{-|n| r / R}$

Finally we arrive at

$$
\begin{aligned}
V(\vec{r}, w)= & -\frac{V_{0}}{2 r R} \sum_{n \in \mathbb{Z}}\left[1+i \theta\left(\frac{1}{r}+\frac{|n|}{R}\right) \partial_{0}\right] \\
& \times e^{-(|n| r-i n w) / R} .
\end{aligned}
$$

Equation (33) can also be expanded using the Fourier series as follows:

$$
\begin{aligned}
V(\vec{r}, w)= & -\frac{V_{0}}{2 r R} \sum_{n \in \mathbb{Z}}\left[1+i \theta\left(\frac{1}{r}+\frac{|n|}{R}\right) \partial_{0}\right] \\
& \times e^{-(|n| r-i n w) / R},
\end{aligned}
$$

and the radial equation takes the form

$\left[\frac{\mathrm{d}^{2}}{\mathrm{~d} r^{2}}+\frac{2}{r} \frac{\mathrm{d}}{\mathrm{d} r}-\frac{n^{2}}{R^{2}}+\alpha^{2}+\frac{u}{r}-\frac{v}{r^{2}}\right] \psi_{n}(r)=0$,

where $\alpha^{2}=\frac{2 m E}{\hbar^{2}} ; v=\lambda_{4}-\frac{m V_{0} E \theta}{\hbar^{3} R}, u=\frac{m V_{0}}{\hbar^{2} R}$. The solution of this equation leads to the same results as given in (27) and (31).

\section{Discussion and conclusion}

In this paper we have found that the noncommutativity of spacetime can help to compute the exact expression of the energy spectrum of the hydrogen atom in $(4+1)$ dimensions with one compactified extra dimension. Unfortunately, it is clear that this method cannot be used in higher dimensions. To be more precise, let us consider the particular case where $D=6$, the compactified one dimension $x^{6}$ gives the potential

$$
\begin{aligned}
V(\vec{r}, w)= & -\frac{q_{e}^{2}}{(2 R r)^{2}}\left(\frac{R}{r} \frac{\sinh (r / R)}{\cosh (r / R)-\cos (w / R)}\right. \\
& \left.+\frac{\cosh (r / R) \cos (w / R)-1}{[\cosh (r / R)-\cos (w / R)]^{2}}\right) \\
& +\frac{i \theta q_{e}^{2}}{8 r^{4} R^{3}(\cos (w / R)-\cosh (r / R))^{3}} \\
& \times\left[\left(r^{2}+3 R^{2}\right) \sinh (r / R) \cos (2 w / R)\right. \\
& +\cos (w / R)\left(\left(r^{2}-6 R^{2}\right) \sinh (2 r / R)\right. \\
& +3 r R(\cosh (2 r / R)+3)) \\
& +3 \sinh (r / R)\left(R^{2} \cosh (2 r / R)+2 R^{2}-r^{2}\right) \\
& -3 r R \cosh (r / R)(\cos (2 w / R)+3)] \partial_{0} .
\end{aligned}
$$

This relation can be expanded in a Fourier series as

$$
\begin{aligned}
V(\vec{r}, w)= & -\frac{q_{e}^{2}}{(2 R r)^{2}} \sum_{n \in \mathbb{Z}}\left[\left(\frac{R}{r}+|n|\right)\right. \\
& \left.+i \theta\left(\frac{3 R}{r^{2}}+\frac{3|n|}{r}+\frac{n^{2}}{R}\right) \partial_{0}\right] e^{-|n| r / R} e^{i n w / R}
\end{aligned}
$$

in which the radial part of the Schrödinger equation becomes

$$
\left(\frac{\mathrm{d}^{2}}{\mathrm{~d} r^{2}}+\frac{2}{r} \frac{\mathrm{d}}{\mathrm{d} r}-\frac{\lambda_{4}}{r^{2}}-\frac{n^{2}}{R^{2}}+\alpha^{2}+\frac{2 m q_{e}^{2}}{4 \hbar^{2} r^{3} R}+\frac{3 m q_{e}^{2} E \theta}{2 \hbar^{3} r^{4} R}\right) \psi_{n}(r)=0 .
$$

The solution of this equation is not yet understood. Surprisingly, we have also shown that, despite this noncommutativity, the energy spectrum does not depend on the deformation parameter $\theta$ and therefore might be considered as the energy solution of commutative space, with one compactified extra dimension, solved in [17] and [18]. Finally let us mention that in the case of higher dimensions higher than $4+1$ the compactified several extra dimensions may be considered.

Let us examinate the case of the Klein-Gordon (KG) equation:

$$
\begin{aligned}
& \left(i \hbar \partial_{0}-V\left(\vec{r}_{n c}\right)\right)^{2} \psi\left(\vec{r}_{n c}, t\right) \\
& \quad=m^{2} c^{4} \psi\left(\vec{r}_{n c}, t\right)-\hbar^{2} c^{2} \Delta \psi\left(\vec{r}_{n c}, t\right),
\end{aligned}
$$

where the potential $V\left(\vec{r}_{n c}\right)$ is

$V\left(\vec{r}_{n c}\right)=-\wp \frac{\hbar c}{\left|\vec{r}_{n c}\right|^{D-2}}$ 
$\wp$ is related to the fine structure constant and $c$ is the light speed. For $D=4$, by taking into account the fact that $\vec{r}_{n c}=\vec{r}-i \vec{\theta} \partial_{0}$, and writing the extra dimension $x^{4}=$ $w-2 \pi R k, k \in \mathbb{Z}$, we get

$$
\begin{aligned}
V(r, w)= & -\wp \hbar c \sum_{n=-\infty}^{\infty}\left\{\frac{1}{r^{2}+(w-2 \pi n R)^{2}}\right. \\
& \left.+\frac{2 i r \theta}{\left(r^{2}+(w-2 \pi n R)^{2}\right)^{2}} \partial_{0}\right\} .
\end{aligned}
$$

The left hand side of Eq. (47), using (49), gives

$$
\begin{aligned}
& \left(i \hbar \frac{\partial}{\partial t}-V(r, w)\right)^{2} \psi(\vec{r}, t) \\
& =\left[-\hbar^{2} \partial_{0}^{2}+\wp \hbar^{2} c\left(2 i A(r, w) \partial_{0}+3 \theta \frac{\partial A(r, w)}{\partial r} \partial_{0}^{2}\right)\right. \\
& \left.\quad+\wp^{2} \hbar^{2} c^{2}\left(A^{2}(r, w)-i \theta \frac{\partial A^{2}(r, w)}{\partial r} \partial_{0}\right)\right] \psi(\vec{r}, t)
\end{aligned}
$$

where $A(r, w)$ is written as

$$
A(r, w)=\frac{1}{2 R r} \frac{\sinh (r / R)}{\cosh (r / R)-\cos (w / R)},
$$

with the Fourier series

$A(r, w)=\frac{1}{2 r R} \sum_{n \in \mathbb{Z}} e^{i n w / R} e^{i n w / R}$.

After some technical handling, we can show that the quantity $A^{2}(r, w)$ is expanded as

$A^{2}(r, w)=\frac{1}{(2 R r)^{2}} \sum_{n \in \mathbb{Z}}\left[|n|+\operatorname{coth}\left(\frac{r}{R}\right)\right] e^{-\frac{|n| r}{R}} e^{\frac{i n w}{R}}$.

By replacing (52) and (53) in (50) and separating variables as $\psi(\vec{r}, t)=\Psi(\vec{r}) e^{-i E t / \hbar}$ and $\Psi(\vec{r})=\Psi(r) \mathcal{Y}_{\ell}^{(3)}$ such that $\hat{l}^{2} \mathcal{Y}_{\ell}{ }^{(3)}=\ell(\ell+2) \mathcal{Y}_{\ell}{ }^{(3)}=\lambda_{4} \mathcal{Y}_{\ell}{ }^{(3)}$ we find the radial equation:

$$
\left[\frac{1}{r^{2}} \mathrm{~d}\left(r^{2} \frac{\mathrm{d}}{\mathrm{d} r}\right)+V_{\mathrm{eff}}(r)+\frac{E^{2}}{\hbar^{2} c^{2}}-\frac{m^{2} c^{2}}{\hbar^{2}}-\frac{n^{2}}{R^{2}}\right] \Psi_{n}(r)=0,
$$

where the effective potential $V_{\text {eff }}(r)$ is

$$
\begin{aligned}
V_{\mathrm{eff}}(r)= & -\frac{\lambda_{4}}{r^{2}}+\frac{3 \theta \wp E^{2}}{2 r^{2} R \hbar^{2} c}+\frac{\wp E}{r R \hbar c}+\frac{\wp^{2}}{(2 R r)^{2}} \operatorname{coth}(r / R) \\
& +\frac{\theta \wp^{2} E}{(2 R r)^{2} \hbar}\left(\frac{2}{r} \operatorname{coth}(r / R)+\frac{r}{\sinh ^{2}(r / R)}\right) .
\end{aligned}
$$

Equation (54) can be solved numerically using the approximation method (Fig. 1). Consider the Taylor expansion of $V_{\text {eff }}$ by using the fact that

$\operatorname{coth}(r / R)=\frac{R}{r}+\frac{r}{3 R}+\cdots$
$\sinh (r / R)=\frac{r}{R}+\cdots$.

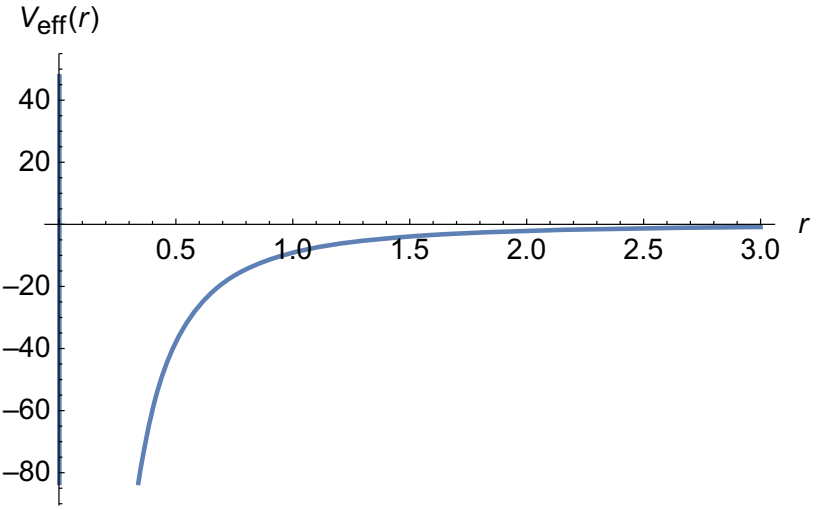

Fig. 1 Plot of the potential $V_{\text {eff }}(r)$, with $R=0.01, \theta=0.01, \hbar=$ $c=1, \wp=1 / 137, E=1, \ell=1$

Then (54) becomes

$$
\frac{\mathrm{d}^{2} \Psi_{n}(r)}{\mathrm{d} r^{2}}+\frac{2}{r} \frac{\mathrm{d} \Psi_{n}(r)}{\mathrm{d} r}+\sum_{j=0}^{4} \frac{b_{j}}{r^{j}} \Psi_{n}(r)=0,
$$

with $b_{4}=\frac{\theta \wp^{2} E}{2 R \hbar}, b_{3}=\frac{\theta \wp^{2} E}{4 \hbar}+\frac{\wp^{2}}{4 R}, b_{2}=\frac{\theta \wp^{2} E}{6 R^{3} \hbar}+\frac{3 \theta \wp E^{2}}{2 R \hbar^{2} c}-$ $\lambda_{4}, b_{1}=\frac{\wp^{2}}{12 R^{3}}+\frac{\wp E}{R \hbar c}, b_{0}=\frac{E^{2}}{\hbar^{2} c^{2}}-\frac{m^{2} c^{2}}{\hbar^{2}}-\frac{n^{2}}{R^{2}}$.

We shall first examine the wave functions $\Psi_{n}(r)$ in the asymptotic range, $r \rightarrow \infty$. The potential $V_{\text {eff }}(r)$ vanishes, in this limit, i.e.,

$V_{\text {eff }}\left(r_{\infty}\right) \rightarrow 0$.

In the region $r_{\infty}$ Eq. (57) gives the solution of the form

$\Psi_{n}^{\infty}(r)=U \frac{e^{-\sqrt{-b_{0}} r}}{r}, \quad U \in \mathbb{R}$.

The general solution of Eq. (57) takes the form

$\Psi_{n}(r)=U(r) \frac{e^{-\sqrt{-b_{0}} r}}{r}$,

where $U(r)$ satisfies the differential equation

$U^{\prime \prime}(r)-2 \sqrt{-b_{0}} U^{\prime}(r)+\sum_{j=1}^{4} \frac{b_{j}}{r^{j}} U(r)=0$.

The investigation of the numerical solution of this equation can be made in forthcoming work.

Acknowledgments DOS research at Max-Planck Institute is supported by the Alexander von Humboldt foundation. The authors are grateful to the referee for his useful comments that allowed them to improve the paper.

Open Access This article is distributed under the terms of the Creative Commons Attribution 4.0 International License (http://creativecomm ons.org/licenses/by/4.0/), which permits unrestricted use, distribution, and reproduction in any medium, provided you give appropriate credit to the original author(s) and the source, provide a link to the Creative Commons license, and indicate if changes were made. Funded by SCOAP $^{3}$. 


\section{References}

1. H.S. Snyder, Quantized space-time. Phys. Rev. 71, 38 (1947). doi:10.1103/PhysRev.71.38

2. A. Connes, Noncommutative geometry. ISBN-9780121858605

3. A. Connes, J. Lott, Particle models and noncommutative geometry (expanded version). Nucl. Phys. Proc. Suppl. 18B, 29 (1991)

4. R.J. Szabo, Quantum field theory on noncommutative spaces. Phys. Rep. 378, 207 (2003). doi:10.1016/S0370-1573(03)00059-0. arXiv:hep-th/0109162

5. H. Grosse, R. Wulkenhaar, Commun. Math. Phys. 256, 305 (2005). doi:10.1007/s00220-004-1285-2. arXiv:hep-th/0401128

6. S. Doplicher, K. Fredenhagen, J.E. Roberts, The quantum structure of space-time at the Planck scale and quantum fields. Commun. Math. Phys. 172, 187 (1995). arXiv:hep-th/0303037

7. S. Majid, Meaning of noncommutative geometry and the Planck scale quantum group. Lect. Notes Phys. 541, 227 (2000). arXiv:hep-th/0006166

8. A.F. Ferrari, H.O. Girotti, M. Gomes, Lorentz symmetry breaking in the noncommutative Wess-Zumino model: one loop corrections. Phys. Rev. D 73, 047703 (2006). arXiv:hep-th/0510108

9. S.'I. Imai, N. Sasakura, Scalar field theories in a Lorentz invariant three-dimensional noncommutative space-time. JHEP 0009, 032 (2000). arXiv:hep-th/0005178

10. N. Seiberg, E. Witten, String theory and noncommutative geometry. JHEP 9909, 032 (1999). doi:10.1088/1126-6708/1999/09/032. arXiv:hep-th/9908142

11. J.M. Carmona, J.L. Cortes, J. Gamboa, F. Mendez, Noncommutativity in field space and Lorentz invariance violation. Phys. Lett. B 565, 222 (2003). arXiv:hep-th/0207158

12. R. Jackiw, Physical instances of noncommuting coordinates. Nucl. Phys. Proc. Suppl. 108, 30 (2002). arXiv:hep-th/0110057. [Phys. Part. Nucl. 33, S6 (2002)] [Lect. Notes Phys. 616, 294 (2003)]
13. G. Berrino, S.L. Cacciatori, A. Celi, L. Martucci, A. Vicini, Noncommutative electrodynamics. Phys. Rev. D 67, 065021 (2003). arXiv:hep-th/0210171

14. M. Moumni, A. BenSlama, S. Zaim, Spectrum of hydrogen atom in space-time non-commutativity. Afr. Rev. Phys. 7, 0010 (2012). arXiv:1003.5732 [hep-ph]

15. M. Moumni, A. BenSlama, S. Zaim, A New limit for the noncommutative space-time parameter. J. Geom. Phys. 61, 151 (2011). doi:10.1016/j.geomphys.2010.09.010. arXiv:0907.1904 [hep-ph]

16. H.T. Elze, Emergent discrete time and quantization: relativistic particle with extra dimensions. Phys. Lett. A 310, 110 (2003). doi:10. 1016/S0375-9601(03)00340-2. arXiv:gr-qc/0301109

17. M. Bures, Energy spectrum of the hydrogen atom in a space with one compactified extra dimension, $\mathbb{R}^{3} \times S^{1}$. Ann. Phys. 363, 354 (2015). doi:10.1016/j.aop.2015.10.004. arXiv:1505.08100 [quant$\mathrm{ph}]$

18. M. Bures, P. Siegl, Hydrogen atom in space with a compactified extra dimension and potential defined by Gauss' law. Ann. Phys. 354, 316 (2014). doi:10.1016/j.aop.2014.12.017. arXiv:1409.8530 [quant-ph]

19. M. Chaichian, M.M. Sheikh-Jabbari, A. Tureanu, Noncommutativity of space-time and the hydrogen atom spectrum. Eur. Phys. J. C 36, 251 (2004). doi:10.1140/epjc/s2004-01886-1. arXiv:hep-th/0212259

20. M. Chaichian, M.M. Sheikh-Jabbari, A. Tureanu, Hydrogen atom spectrum and the Lamb shift in noncommutative QED. Phys. Rev. Lett. 86, 2716 (2001). doi:10.1103/PhysRevLett.86.2716. arXiv:hep-th/0010175 